\title{
Prevalence of prothrombotic polymorphisms in a selected cohort of cryptogenic and noncryptogenic ischemic stroke patients
}

\author{
Rocco Salvatore Calabrò, Paolino La Spina ${ }^{1}$, Salvatore Serra ${ }^{1}$, Angelina Laganà ${ }^{1}$, Paolo Postorino ${ }^{1}$, Rodolfo Savica ${ }^{1}$ \\ Corrado Mammì', Carmelo Laganà ${ }^{2}$, Rosa Musolino ${ }^{1}$ \\ IRCCS Centro Neurolesi "Bonino-Pulejo”, ${ }^{1}$ Dipartimento di Neuroscienze, Policlinico Universitario, Messina, ${ }^{2}$ U.O. Genetica, \\ Ospedali Riuniti di Reggio di Calabria, Italy
}

\author{
Address for correspondence: \\ Dr. Rocco Salvatore Calabrò, \\ IRCCS Centro Neurolesi, \\ "Bonino-Pulejo", S.S. 113 via \\ Palermo, Cda Casazza, \\ 98124 Messina, Italy. \\ E-mail: salbro77@tiscali.it
}

PMID: 19934566

DOI: $10.4103 / 0028-3886.57819$

\begin{abstract}
Ischemic stroke is a complex multifactorial disease and approximately $30 \%$, especially in the young, are cryptogenic. In some of the patients with cryptogenic ischemic stroke the underlying risk factor may be a prothrombotic state. We studied 101 patients with ischemic stroke under 55 years of age. All the patients underwent an extensive diagnostic evaluation to determine the cause of stroke. Common variations in the genes encoding factor $\mathrm{V}$, prothrombin, 5,10-methylenetetrahydrofolate reductase, plasminogen activator inhibitor-1, and human platelet alloantigens- 1 were evaluated. Of the 101 patients with ischemic stroke, 28 patients had cryptogenic ischemic stroke. At least one of the different genetic polymorphisms investigated was present in $44 \%$ patients in the total group and in $48 \%$ of patients with cryptogenic ischemic stroke. In this study population under 55 years of age there was no significant difference in the prevalence of various genetic polymorphisms, factor $\mathrm{V}$, prothrombin, 5,10-methylenetetrahydrofolate reductase, plasminogen activator inhibitor-1, and human platelet alloantigens) in patients with cryptogenic ischemic stroke and in patients with ischemic stroke of determined cause.
\end{abstract}

Key words: Cryptogenic stroke, juvenile ischemic stroke, prothrombotic genetic polymorphisms

\section{Introduction}

Ischemic stroke is a complex multifactorial disease and approximately $30 \%$ strokes are cryptogenic, especially in the young adults..$^{[1]}$ In the absence of common vascular risk factors, prothrombotic states should be considered as the possible cause of ischemic stroke and should be investigated. Common variants in several genes contribute to the risk of ischemic stroke and a significant association is found with factor V (FV) Leiden, methylenetetrahydrofolate reductase (MTHRF) C677T, prothrombin (PT) G20210A, and angiotensin-converting enzyme insertion/deletion. ${ }^{[2]}$ We studied the prevalence of various common genetic polymorphisms in patients with cryptogenic ischemic stroke under 55 years of age and compared the prevalence in patients with ischemic stroke of determined cause.

\section{Materials and Methods}

This was hospital-based prospective study of 101 patients with ischemic stroke under 55 years of age between April 2003 and December 2005. Our diagnostic investigations included physical and neurologic examination, computed tomography (CT) and/or magnetic resonance imaging (MRI) of the brain, carotid and intracranial Doppler ultrasound, transthoracic 
and/or transesophageal echocardiography with bubbles contrast, and a complete hematological screening. Stroke was classified using trial of ORG 10172 in acute stroke treatment (TOAST) criteria. Subjects were grouped to one of the two categories: Cryptogenic stroke or stroke of determined cause (non cryptogenic). Factor V, PT G20210A, MTHFR, human platelet alloantigens (HPA-1)-also identified as PLA1/ A2-polymorphism of the platelet glycoprotein receptor (IIb/IIIa), and plasminogen activator inhibitor-1 (PAI-1) mutations were detected using specific standard analysis techniques. The statistical analysis between the two groups was made through the Chisquare and Fisher's exact tests. A $P$-value $<0.05$ was considered significant.

\section{Results}

Of the 101 patients recruited in the study, 54 were females and the age was $45.7 \pm 8.16$ years (range 16-55). All the patients had CT and MRI was done in 67 patients. The stroke distribution was anterior circulation in 80 patients and posterior circulation in 21. According to TOAST criteria the stroke subtypes were: Cryptogenic stroke in 28, small vessel disease in 21; cardioembolic in 19, large vessel disease in 16, and undetermined in 17. Hyperhomocysteinemia was the most common risk factor $(41.5 \%)$ and was statistically related to the MTHFR mutation $(P<0.05)$. Hypertension was present in only 22 patients. Cardiac lesion was identified in 31. Postive family history cerebrovascular diseases was present in 25 patients. Of the 101 patients, in 44 patients at least one of the genetic polymorphisms investigated was positive, more so in young patients. Twenty patients were homozygous for MTHFR C677T, 14 were carriers of PAI polymorphism (9 were heterozygous and 5 homozygous), 6 were heterozygous for FV Leiden, 7 were heterozygous for PT mutation, and 9 for HPA-1b [Table 1]. The statistical analysis between stroke of determined cause and cryptogenic stroke showed no significant differences in the prevalence of genetic polymorphisms investigated.

\section{Discussion}

Ischemic stroke is thought to have a polygenic basis and genetic predisposition and cerebral ischemia may result from an additive effect of several genes or from the interactions of genes with environmental or behavioural factors. ${ }^{[3]}$

The strength of the present study is the accurate and extensive diagnostic evaluations in all the 101 patients so as to categorize the patients accurately into various subtypes by TOAST criteria. In our study, cryptogenic

\begin{tabular}{l|ccc}
\hline \multicolumn{4}{l}{$\begin{array}{l}\text { Table 1: Comparison of the prothrombotic genetic polymorphisms } \\
\text { between stroke of determined and undetermined causes }\end{array}$} \\
\hline $\begin{array}{l}\text { Genetic } \\
\text { polymorphisms }\end{array}$ & $\begin{array}{c}\text { Stroke of } \\
\text { determined } \\
\text { causes (\%) }\end{array}$ & $\begin{array}{c}\text { Stroke of } \\
\text { undetermined } \\
\text { causes (\%) }\end{array}$ & $P$ value \\
\hline MTHFR C677T & $15(20.83)$ & $5(17.24)$ & $>0.5^{*}$ \\
FV Leiden & $3(4.17)$ & $3(10.34)$ & $>0.5^{*}$ \\
PT G20210A & $4(5.56)$ & $3(10.34)$ & $>0.5^{*}$ \\
PAI-1 & $8(11.11)$ & $6(20.69)$ & $>0.5^{*}$ \\
HPA-1 & $8(11.11)$ & $1(3.45)$ & $>0.2^{*}$ \\
\hline
\end{tabular}

*Statistically non-significant; MTHFR - 5,10-Methylenetetrahydrofolate reductase; FV - Factor V Leiden; PT - Prothrombin; PAI - Plasminogen activator inhibitor-1; HPA - Human platelet alloantigens

strokes accounted for about $28 \%$ which is much lower than the incidence reported previously. ${ }^{[4]}$ In our study there were no patients with two or more causes. The most common risk factor was hyperhomocysteinemia and the total serum homocysteine level correlated with the presence of the MTHRF C677T polymorphism and to the folate and B12 intake, similar to the observations in other studies. ${ }^{[5]}$ In the present study, we did not find any statistically significant difference in the prevalence of various genetic polymorphisms investigated between patients with cryptogenic stroke and patients with stroke of determined cause. These findings suggest that presence of genetic thrombophilic state per se may not represent an independent risk factor for ischemic stroke and it could be an important marker of ischemic events. The major limitations of our study include small sample size, no control group, and hospital-based study population. Further multicentric studies using large sample sizre are needed to assess accurately individual susceptibility to cerebral infarction and also to realize a specific preventive strategy, both in young and middle-aged patients with ischemic stroke.

\section{References}

1. Austin H, Chimowitz MI, Hill HA, Chaturvedi S, Wechsler LR, Wityk RJ, et al. Cryptogenic stroke in relation to genetic variation in clotting factors and other genetic polymorphisms among young men and women. Stroke 2002;33:2762-8.

2. Casas JP, Hingorani AD, Bautista LE, Sharma P. Meta-analysis of genetic studies in ischemic stroke. Arch Neurol 2004;61:1652-61.

3. Pezzini A, Grassi M, Del Zotto E, Archetti S, Spezi R, Vergani V, et al. Cumulative effect of predisposing genotypes and their interaction with modifiable factors on the risk of ischemic stroke in young adults. Stroke 2005;36:533-9.

4. Naess H, Nyland HI, Thomassen L, Aarseth J, Myhr KM. Etiology of and risk factors for cerebral infarction in young adults in western Norway: A population-based case-control study. Eur J Neurol 2004;11:25-30.

5. Kelly PJ, Rosand J, Kistler JP, Shih VE, Silveira S, Plomaritoglou A, et al. Homocysteine, MTHFR 677CT polymorphism, and risk of ischemic stroke: Results of a meta-analysis. Neurology 2002;59:529-36.

Accepted on 17-03-2009

Source of Support: Nil, Conflict of Interest: None declared. 